Article

\title{
Designing a New Ni-Mn-Sn Ferromagnetic Shape Memory Alloy with Excellent Performance by Cu Addition
}

\author{
Kun Zhang ${ }^{1}$, Xiaohua Tian ${ }^{2, *}$, Changlong Tan ${ }^{1,2}$, Erjun Guo ${ }^{1}$, Wenbin Zhao ${ }^{2}$ and Wei Cai ${ }^{3}$ \\ 1 School of Materials Science and Engineering, Harbin University of Science and Technology, \\ Harbin 150080, China; kunzhang@hrbust.edu.cn (K.Z.); changlongtan@hrbust.edu.cn (C.T.); \\ guoerjun@126.com (E.G.) \\ 2 School of Science, Harbin University of Science and Technology, Harbin 150080, China; \\ Wenbin_zhao1@163.com \\ 3 School of Materials Science and Engineering, Harbin Institute of Technology, Harbin 150001, China; \\ weicai@hit.edu.cn \\ * Correspondence: xiaohuatian@hrbust.edu.cn; Tel.: +86-451-8639-0788
}

Received: 3 February 2018; Accepted: 24 February 2018; Published: 28 February 2018

\begin{abstract}
Both magnetic-field-induced reverse martensitic transformation (MFIRMT) and a high working temperature are crucial for the application of Ni-Mn-Sn magnetic shape memory alloys. Here, by first-principles calculations, we demonstrate that the substitution of $\mathrm{Cu}$ for $\mathrm{Sn}$ is effective not only in enhancing the MFIRMT but also in increasing martensitic transformation, which is advantageous for its application. Large magnetization difference $(\Delta M)$ in Ni-Mn-Sn alloy is achieved by $\mathrm{Cu}$ doping, which arises from the enhancement of magnetization of austenite due to the change of $\mathrm{Mn}-\mathrm{Mn}$ interaction from anti-ferromagnetism to ferromagnetism. This directly leads to the enhancement of MFIRMT. Meanwhile, the martensitic transformation shifts to higher temperature, owing to the energy difference between the austenite $L 2_{1}$ structure and the tetragonal martensite $L 1_{0}$ structure increases by $\mathrm{Cu}$ doping. The results provide the theoretical data and the direction for developing a high temperature magnetic-field-induced shape memory alloy with large $\Delta M$ in the Ni-Mn-Sn Heusler alloy system.
\end{abstract}

Keywords: Ni-Mn-Sn; ferromagnetic shape memory alloys; martensitic transformation; magnetic properties; first-principle calculation; $\mathrm{Cu}$ addition

\section{Introduction}

Owing to the coupling of structure and magnetism, the ferromagnetic shape memory alloys (FSMAs) have attracted considerable attention [1-6]. The magnetic shape memory arises from a magnetic-field-induced rearrangement of martensite variants in Ni-Mn-Ga [7]. Based on this mechanism, because the driving force is limited by the anisotropy energy, the output stress induced by the external magnetic field is restricted [3]. Different from Ni-Mn-Ga FSMAs, Ni-Mn- $X(X=\mathrm{In}$, $\mathrm{Sn}$, and $\mathrm{Sb}$ ) FSMAs can obtain larger output stress resulting from the magnetic-field-induced transformation [8]. The shift of the reverse martensitic transformation temperature induced by magnetic field, $\Delta T$, is given by the Clausius-Clapeyron equation:

$$
\frac{d B}{d T}=\frac{\Delta S}{\Delta M} \text { and } \Delta T \approx\left(\frac{\Delta M}{\Delta S}\right) \Delta B
$$

where $B$ is the applied magnetic field, and $\Delta M$ and $\Delta S$ are the differences in magnetization and entropy between parent and martensite phase, respectively. Recently, the Mn-rich Ni-Mn-Sn FSMAs have 
stood out due to their remarkable properties such as giant caloric effects [9] and complex magnetic behavior with easily-tunable transformation characteristics by a variation of the composition and doping [7-14]. Most of these fascinating properties are related to the magnetic-field-induced reverse transformation (MFIRT). Unfortunately, practical applications of these alloys are restricted because of the drawback which is the requirement for a high magnetic field. As Equation (1) shows, to use MFIRT, a larger $\Delta M$ is urgently required for the martensitic transformation. Up to now, some efforts have been devoted to enlarge $\Delta M$ in Ni-Mn-based FSMAs, such as tuning the stoichiometric, substituting foreign atoms, and applying external pressure [15-19]. Among these, substituting a small amount of $\mathrm{Co} / \mathrm{Fe}$ for $\mathrm{Ni}$ is an efficient method to enhance the $\Delta M$. This is mainly due to the local ferromagnetic structure achieved in the antiferromagnetic matrix and the magnetization of the high temperature phase which was effectively enhanced [20]. The disadvantage of Co doping is that it makes martensitic transformation (MT) temperature too low, even below room temperature. However, the working temperature of Ni-Mn-Sn FSMAs is usually at room temperature or at higher temperatures. Thus, developing the Ni-Mn-Sn FSMAs with a large $\Delta M$ and high MT temperature is essential.

In Ni-Mn-Sn FSMAs, a fourth element addition normally seeks to enhance the magnetic properties, although it can also modify the structure. $\mathrm{Cu}$, for instance, has been shown to have an important effect on the properties of Ni-Mn-Sn compounds. Castillo-Villa et al. have reported improved refrigeration capacity in $\mathrm{Ni}-\mathrm{Mn}-\mathrm{Sn}-\mathrm{Cu}$ polycrystalline through the magnetocaloric and elastocaloric combination [21]. Lately, Li et al. shows that $\mathrm{Ni}_{44} \mathrm{Mn}_{41} \mathrm{Sn}_{11} \mathrm{Cu}_{4}$ exhibits a giant adiabatic cooling of $-8 \mathrm{~K}$ at small transformation strains of $1.3 \%$. Ni-Mn-Sn-Cu alloys are proposed as a candidate for the elastocaloric materials [22]. As for the martensitic transformation, Wang et al. reported that Mn substitution by $\mathrm{Cu}$ increased the martensitic transformation (MT) temperature as well as the magnetocaloric effect [23], while other researchers found that the replacement of $\mathrm{Ni}$ by $\mathrm{Cu}$ atoms can generate an obvious martensitic transformation temperature change to a lower temperature [24]. So far, numerous works have focused on adjusting the transformation temperatures or improving the magnetic properties. However, research has rarely been carried out to improve the $\Delta M$ and MT temperature at the same time. Thus, we expect that doping proper $\mathrm{Cu}$ in the Ni-Mn-Sn FSMAs can simultaneously enhance the $\Delta M$ and improve MT temperature.

However, due to the restrictions of a long experimental period, limited scope, and high costs, experimental studies have limitations when designing a new material. Before the experiment, calculation by the first principle plays an important role in predicting new ferromagnetic shape memory alloys. The theoretical calculation can simulate substitution or addition elements with any desired composition and accordingly select the most promising one, which can provide useful guidance for experiments. Up to this day, first-principle calculations have been successfully used to calculate the electronic structure, formation energy and magnetic structure of the off-stoichiometric Ni-Mn based FSMAs. Meanwhile, the calculated results are consistent with the experimental results. Thus, by first-principle calculation, we expect to design a new FSMAs material with excellent performance through $\mathrm{Cu}$ doping.

In the present paper, the electronic structure and magnetic properties of $\mathrm{Ni}_{16} \mathrm{Mn}_{12} \mathrm{Sn}_{4-x} \mathrm{Cu}_{x}$ alloys $(x=0,1,2,3$, and 4$)$ have been calculated by the first principle. The influence of $\mathrm{Cu}$ doping on phase transformation temperature, Curie temperature and magnetic structure was clarified. This work can help to understand the phase transformation deeply and design new Ni-Mn-Sn alloys with excellent properties by doping another element. Calculated results showed that the substitution of $\mathrm{Cu}$ for $\mathrm{Sn}$ is effective not only in enhancing the MFIRMT, but also in increasing martensitic transformation. The results provide the theoretical data and the direction for developing high temperature magnetic-field-induced shape memory alloy in the Ni-Mn-Sn Heusler alloy system.

\section{Materials and Methods}

The Heusler alloys $\left(X_{2} Y Z\right)$ studies here possess a $L 2_{1}$ structure that consists of four interpenetrating face-centered-cubic (fcc) sublattices with origin at fractional positions, $(0.25,0.25$, 
$0.25),(0.75,0.75,0.75),(0.5,0.5,0.5)$, and $(0,0,0)$. For the conventional Heusler alloy structure, the first two sub-lattices are occupied by $X$ atom and the third by $Y$ and fourth by $Z$ atom. In total, there are 16 atoms in the model, as shown in Figure 1a. The stoichiometrical $\mathrm{Ni}_{8} \mathrm{Mn}_{4} \mathrm{Sn}_{4}$ alloy does not undergo a martensitic phase transition from the cubic Heusler $L 2_{1}$ structure to the non-modulated structure [25]. The previous research about the $\mathrm{Ni}_{8} \mathrm{Mn}_{4+x} \mathrm{Sn}_{4-x}$ alloys have shown that the martensitic transformation can be observed only under the Mn-rich with $x \geq 2$. Here, we have focused on the off-stoichiometrical $\mathrm{Ni}_{8} \mathrm{Mn}_{6} \mathrm{Sn}_{2}$ alloy substitution of $\mathrm{Sn}$ by $\mathrm{Cu}$. Moreover, in order to calculate the influence of different $\mathrm{Cu}$ content more accurately, the $2 \times 1 \times 1$ supercell approach is used for the calculations, as shown in Figure $1 \mathrm{~b}$. In the model, $\mathrm{Mn}_{\mathrm{Mn}}$ represents the $\mathrm{Mn}$ atoms in the $\mathrm{Mn}$ sites, and $\mathrm{Mn}_{\mathrm{Sn}}$ represents $\mathrm{Mn}$ atoms in the Sn sites. In the case of the model of the cubic structure for the austenitic phase of $\mathrm{Ni}_{16} \mathrm{Mn}_{12} \mathrm{Sn}_{4-x} \mathrm{Cu}_{x}$ alloys $(x=1,2,3$, and 4), the same size of the model is used except for some appropriate $\mathrm{Sn}$ atoms being replaced by the doped $\mathrm{Cu}$ atoms. It is worth noting that the simulated structure here is an ordered one, whereas in a real material the $\mathrm{Cu}$ and $\mathrm{Mn}$ ions on the Sn sites would be randomly distributed. This may have an effect on the properties of the material. All calculations have been performed based on the density functional theory, using CASTEP (Cambridge Sequential Total Energy Package) code [26]. The plane-wave cutoff energy is $400 \mathrm{eV}$ which is found to converge the total energy efficiently of the physical models. A $(8,8,8)$ Monkhorst-Pack grid is employed to sample the Brillouin zone. The interaction between ions and electrons is described by ultra-soft pseudopotentials [27]. The spin-polarized generalized gradient approximation is used to describe the exchange correlation energy. The tetragonal martensite has been obtained by calculation of the stability of $L 2_{1}$ austenite with respect to volume-conserving tetragonal distortions. Then, the total energy of both austenite and martensite, electronic structure and magnetic moment have been calculated.

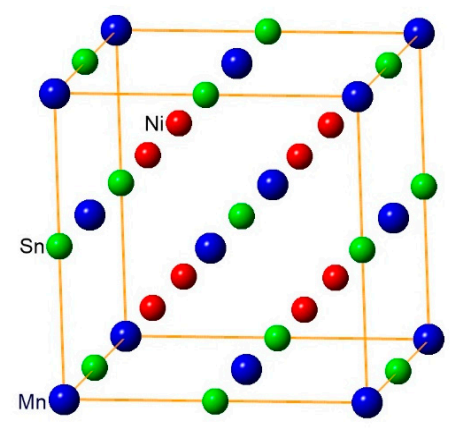

(a)

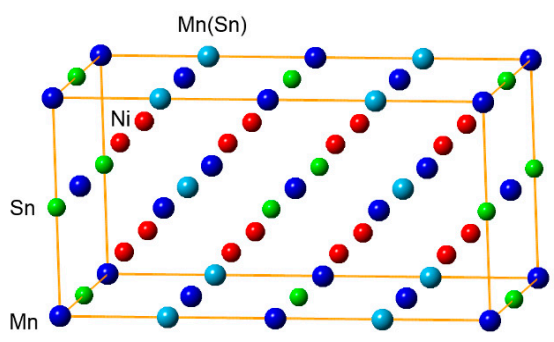

(b)

Figure 1. The unit cell of the cubic structure for the parent of the Ni-Mn-Sn alloy (a) $\mathrm{Ni}_{8} \mathrm{Mn}_{4} \mathrm{Sn}_{4}$ alloy; (b) $\mathrm{Ni}_{16} \mathrm{Mn}_{12} \mathrm{Sn}_{4}$ alloy.

\section{Results and Discussion}

To determine the magnetic ground and theoretical lattice parameter of $\mathrm{Ni}_{16} \mathrm{Mn}_{12} \mathrm{Sn}_{4-x} \mathrm{Cu}_{x}$ alloys $(x=0,1,2,3$, and 4$)$ in the austenitic phase, we performed total energy calculations with spin polarization. We considered two situations where the magnetic moments of the excess to the Mn atoms in the $\mathrm{Sn}$ sites (denoted as $\mathrm{Mn}_{\mathrm{Sn}}$ ) are parallel or anti-parallel to the $\mathrm{Mn}$ atoms in the $\mathrm{Mn}$ sub-lattice site (denoted as $\mathrm{Mn}_{\mathrm{Mn}}$ ). The parallel and antiparallel $\mathrm{Mn}_{\mathrm{Sn}}-\mathrm{Mn}_{\mathrm{Mn}}$ magnetic interactions are denoted as $\mathrm{P}$ and AP states, respectively. Results for the lattice constants of the $\mathrm{Ni}_{16} \mathrm{Mn}_{12} \mathrm{Sn}_{4-x} \mathrm{Cu}_{x}(x=0,1,2,3$, and 4) cubic phases for both the AP and P states are plotted in Figure 2. The calculated energies of the equilibrium lattice constants are $-29,898.73 \mathrm{eV}$ and $-29,898.80 \mathrm{eV}$ for the AP and P states for $x=0$, respectively. For $x=1$, these total energies are $-31,280.29 \mathrm{eV}$ and $-31,280.41 \mathrm{eV}$ for the AP and P states, respectively. In both structures, the AP states are lower on the energy scale and are thus significantly more stable than the $P$ states around the equilibrium lattice parameter for the case of $x=0$ and 1 . This indicates that the magnetic moment of the $\mathrm{Mn}_{\mathrm{Sn}}$ in $\mathrm{Ni}_{16} \mathrm{Mn}_{12} \mathrm{Sn}_{4-x} \mathrm{Cu}_{x}$ alloys ( $x=0$, and 1$)$ cubic 
phase is anti-ferromagnetically coupled to the magnetic moment of $\mathrm{Mn}_{\mathrm{Mn}}$. On the contrary, as for the case of $x=2$, the P states are slightly lower on the energy scale than the AP states with nearly the same energy corresponding to the ground state lattice constant of $\sim 6.0 \AA$. With the further increasing of $\mathrm{Cu}$ content, it is worth noting that, in contrast to the alloys without $\mathrm{Cu}$ doping, the $\mathrm{P}$ state of alloys is evidently more stable than the AP state at equilibrium lattice constant. This result obviously indicates that substitution of proper $\mathrm{Cu}$ for $\mathrm{Sn}$ converts antiferromagnetic austenite to ferromagnetic state in the cubic austenite phase, and hence increases the magnetization of austenite. Meanwhile, it can be seen that the equilibrium lattice constants of $\mathrm{Ni}_{16} \mathrm{Mn}_{12} \mathrm{Sn}_{4-x} \mathrm{Cu}_{x}$ alloys $(x=0,1,2,3$, and 4$)$ decrease with increasing Cu content. Probably because the radius of $\mathrm{Cu}(1.57 \AA)$ is smaller than that of $\mathrm{Sn}(1.72 \AA)$, the addition of $\mathrm{Cu}$ causes the unit-cell volume to shrink.

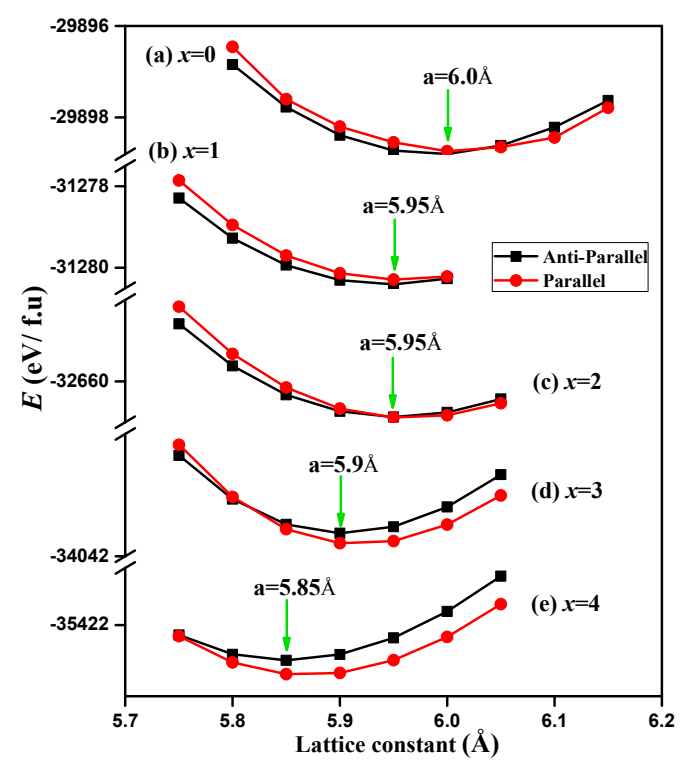

Figure 2. Equation of states of $\mathrm{Ni}_{16} \mathrm{Mn}_{12} \mathrm{Sn}_{4-x} \mathrm{Cu}_{x}$ cubic phase $(x=0,1,2,3$, and 4$)$ for both parallel and antiparallel magnetic interaction.

In order to see the effect of $\mathrm{Cu}$ on martensitic transformation in a Cu-doped Ni-Mn-Sn alloy, the total energy as a function of the tetragonal ratio $\mathrm{c} / \mathrm{a}$ is calculated for both parallel and antiparallel magnetic interactions of $\mathrm{Ni}_{16} \mathrm{Mn}_{12} \mathrm{Sn}_{4-x} \mathrm{Cu}_{x}$ alloys $(x=0,1,2,3$ and 4). Figure 3 shows the total energies of $\mathrm{Ni}_{16} \mathrm{Mn}_{12} \mathrm{Sn}_{4-x} \mathrm{Cu}_{x}$ alloys $(x=0,2$, and 4) with a variation of the tetragonal ratio $\mathrm{c} / \mathrm{a}$, because the curves of the other specimens are similar. For the alloy without $\mathrm{Cu}$ doping as shown in Figure $3 \mathrm{a}$, for structures under the $\mathrm{P}$ state, $\mathrm{Ni}_{16} \mathrm{Mn}_{12} \mathrm{Sn}_{4}$ has only one minimum in the $E$-c/a curve at $\mathrm{c} / \mathrm{a}=0.98$ and the energy difference between $\mathrm{c} / \mathrm{a}=1.00$ is rather small. However, as to the $E-c / a$ curve of structure under AP state, the energy is lower compared with the $E$-c/a curve of structure under P state. This indicates that the AP state is favored energetically for all c/a ranges, which means that the $\mathrm{Mn}_{\mathrm{Sn}}-\mathrm{Mn}_{\mathrm{Mn}}$ magnetic interactions in both austenite and martensite are antiparallel. Also, it is found that, when the structure is under AP state, the energy minimum can be observed at c/a $=1.3$, which corresponds to the $L 1_{0}$ structure of $\mathrm{Ni}_{16} \mathrm{Mn}_{12} \mathrm{Sn}_{4}$. This result shows that the AP austenite phase is unstable against the tetragonal distortion in $\mathrm{Ni}_{16} \mathrm{Mn}_{12} \mathrm{Sn}_{4}$. It undergoes the martensitic transformation from AP austenite phase to AP tetragonal martensite. Due to both austenite and martensite being in the AP state, the $\Delta M$ between the two structural phases is small. This directly leads to the weak MFIRMT in $\mathrm{Ni}_{16} \mathrm{Mn}_{12} \mathrm{Sn}_{4}$ alloy without $\mathrm{Cu}$ addition.

The addition of $\mathrm{Cu}$, from the change of the $E$-c/a curves, shows that the energy behaviors along the variation of the $\mathrm{c} / \mathrm{a}$ do not change. However, $\mathrm{Cu}$ introduction changes the relative stability of the structure. As shown in Figure 3b,c, for the case of $x=2$ and 4 , we find that the P state is more stable than the AP state only around the equilibrium lattice constant for the austenitic phase, as indicated by the blue rectangle. Here, the AP state becomes energetically favorable when the martensitic phase transition 
appears, which indicates that a magneto-structural martensitic transition occurs. Thus, a competition between the $\mathrm{P}$ and $\mathrm{AP}$ states is evidently observed from the $E$-c/a curve of $\mathrm{Ni}_{16} \mathrm{Mn}_{12} \mathrm{Sn}_{0} \mathrm{Cu}_{4}$. In the vicinity of $\mathrm{c} / \mathrm{a}=1$, the structure under $\mathrm{P}$ state is more stable than that of AP state. The energy minimum can be reached at $\mathrm{c} / \mathrm{a}=1$ with respect to the $L 2_{1}$ structure under $\mathrm{P}$ state. With the $\mathrm{c} / \mathrm{a}$ deviating from 1 , the total energy of structure under P state increases. However, for the structure under AP state, the total energy decreases to the value lower than that of $\mathrm{P}$ state and reaches the energy minimum at $\mathrm{c} / \mathrm{a}=1.4$, which corresponds to the martensitic phase $L 1_{0}$ structure under the AP state. Thus, we find that the martensite $L 1_{0}$ structure for each system keeps the AP state, whereas the antiferromagnetic austenite has been tuned to ferromagnetic state by substitution of proper $\mathrm{Cu}$ for $\mathrm{Sn}$.

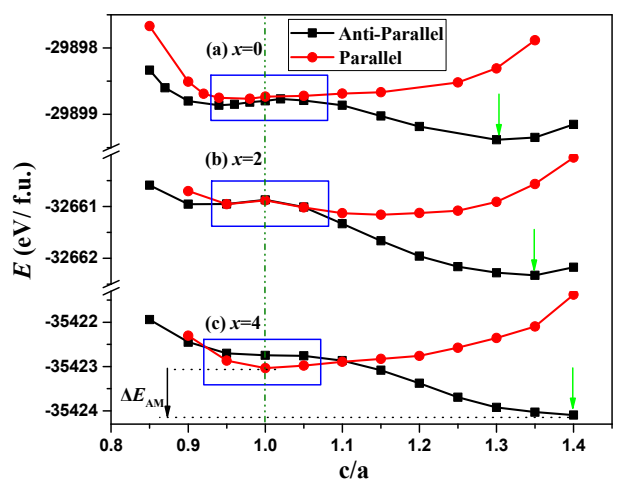

Figure 3. The total energy difference as a function of the tetragonality, $\mathrm{c} / \mathrm{a}$, in the tetragonal structure calculated for both parallel and antiparallel magnetic interaction for $\mathrm{Ni}_{16} \mathrm{Mn}_{12} \mathrm{Sn}_{4-x} \mathrm{Cu}_{x}$ with $x=0,2$, and 4 , respectively. The vertical line located at $\mathrm{c} / \mathrm{a}=1$ denotes the position of the cubic austenite phase.

A good deal of previous research has shown the ferromagnetic activation effect of Co or Fe elements. From the above analysis, we found the same function of $\mathrm{Cu}$ addition in Ni-Mn-Sn alloy. Furthermore, the concentration dependence of magnetic moment per formula unit for $\mathrm{Ni}_{16} \mathrm{Mn}_{12} \mathrm{Sn}_{4-x} \mathrm{Cu}_{x}$ alloys $(x=0,1,2,3$, and 4$)$ is depicted in Figure 4 . In the case of $x=0$ and 1 , the magnetization difference between the austenite and the martensite is very small. With the increasing of $\mathrm{Cu}$ content, in the case of $x=2,3$, and 4, the magnetic moment of austenite was significantly enhanced, while the magnetic moment of martensite changed little. Thus, the magnetization difference between the austenite and the martensite is enlarged evidently. This result is consistent with the conclusion of the E-c/a curves. This should be attributed to a change of magnetic structure of austenite caused by the substitution of proper $\mathrm{Cu}$ for $\mathrm{Ni}$, which turns the Mn-Mn interaction from anti-ferromagnetism to ferromagnetism. Such a high magnetization difference provides a strong driving force for magnetic-field-induced transformation. Thus, a large $\Delta M$ can be obtained by $\mathrm{Cu}$ addition in the Ni-Mn-Sn alloy system. We finished the first step in designing the new material with a large $\Delta M$.

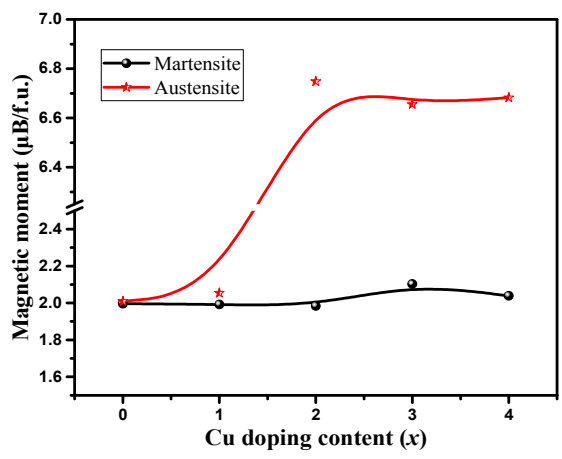

Figure 4. Concentration dependence of magnetic moment per formula unit for $\mathrm{Ni}_{16} \mathrm{Mn}_{12} \mathrm{Sn}_{4-x} \mathrm{Cu}_{x}$ alloys $(x=0,1,2,3$, and 4$)$. 
As ever, researchers have pointed out that the total energy difference between the austenite $L 2_{1}$ structure and the tetragonal martensite $L 1_{0}$ structure $\left(\Delta E_{\mathrm{AM}}=E_{A P(\mathrm{Cub} .)}-E_{A P \text { (Tetra. })}\right)$ can be used to predict the possible martensitic transformation and to estimate the MT temperature, approximately [28]. Generally, the larger energy difference implies a higher MT temperature. In order to clarify the effect of $\mathrm{Cu}$ element on martensitic transformation, the $\Delta E_{\mathrm{AM}}$ (as indicated by the black arrow in Figure 4) is calculated. Figure 5 shows the $\Delta E_{\text {AM }}$ per formula unit for $\mathrm{Ni}_{16} \mathrm{Mn}_{12} \mathrm{Sn}_{4-x} \mathrm{Cu}_{x}$ alloys $(x=0,1,2,3$, and 4). Firstly, we can see that $\Delta E_{\mathrm{AM}}$ increases markedly with a little amount of $\mathrm{Cu}$ doping. In the case of $x=2$, the $\Delta E_{\mathrm{AM}}$ increases to the maximum. As $x$ further increases to 3 and 4 , the $\Delta E_{\mathrm{AM}}$ gradually decreases, which is also higher than that of alloy without $\mathrm{Cu}$ addition. Thus, due to a larger $\Delta E_{\mathrm{AM}}$ corresponds to a higher TM temperature, the results show that the TM temperature first increases and then decreases with the increasing of $\mathrm{Cu}$ content. Proper substitution of $\mathrm{Cu}$ for $\mathrm{Sn}$ can increase the MT temperature. Now, we finished the second step to design a new material with high MT temperature. These results provide origin for the design of high-temperature magnetic shape memory alloys with low magnetic field.

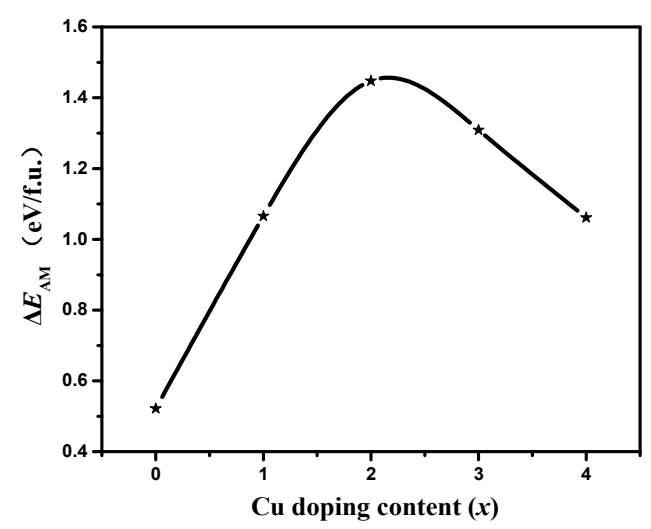

Figure 5. The energy difference between austenite $L 2_{1}$ structure and the tetragonal martensite $L 1_{0}$ structure $\left(\Delta E_{\mathrm{AM}}\right)$ per formula unit for $\mathrm{Ni}_{16} \mathrm{Mn}_{12} \mathrm{Sn}_{4-x} \mathrm{Cu}_{x}$ alloys $(x=0,1,2,3$, and 4$)$.

In practice, elevating the Curie temperature $\left(T_{\mathrm{c}}\right)$ while keeping the martensitic transformation temperature high is essential. Since Ni-Mn-Sn-Cu alloys satisfy the Stone condition of ferromagnetism [29], the $T_{\mathrm{c}}$ can be taken to be proportional to the total energy difference between the paramagnetic and the ferromagnetic state $\left(\Delta E_{\mathrm{tot}}\right)$ [30]. According to the Heisenberg model and the molecular field theory, the link between $\Delta E_{\text {tot }}$ and $T_{\mathrm{c}}$ is given by

$$
\Delta E_{\text {tot }}=-k_{\mathrm{b}} T_{\mathrm{c}} \xi
$$

where $\xi$ represents the ratio $M / M_{0}$ of the magnetic moment $M$ at $T \neq 0 \mathrm{~K}$ and the equilibrium magnetic moment $M_{0}$ at $T=0 \mathrm{~K}$. A number of researchers predict the $T_{\mathrm{c}}$ by using the Equation (2). Though the predicted values have some deviations compared with the experimental values, only from the total energy difference results, we can still draw the conclusion that the $T_{\mathrm{c}}$ can be taken to be proportional to the $\Delta E_{\text {tot. }}$. In order to investigate the effect of $\mathrm{Cu}$ introduction on the $T_{\mathrm{c}}$, the total energies of both ferromagnetic and paramagnetic cubic phase of $\mathrm{Ni}_{16} \mathrm{Mn}_{12} \mathrm{Sn}_{4-x} \mathrm{Cu}_{x}$ alloys $(x=0,1$, 2,3 , and 4) were calculated. The calculated results show that the total energy of paramagnetic cubic phase is much higher than that of the ferromagnetic one at all the compositions studied, which is consistent with the fact that the paramagnetic cubic is the high-temperature phase. Figure 6 shows the total energy difference between the paramagnetic austenite and ferromagnetic austenite per formula unit for $\mathrm{Ni}_{16} \mathrm{Mn}_{12} \mathrm{Sn}_{4-x} \mathrm{Cu}_{x}$ alloys ( $x=0,1,2,3$, and 4). Obviously, it can be seen that the $\Delta E_{\text {tot }}$ linearly decreases with the increase of $\mathrm{Cu}$ content. This result indicates that $T_{\mathrm{c}}$ of $\mathrm{Ni}_{16} \mathrm{Mn}_{12} \mathrm{Sn}_{4-x} \mathrm{Cu}_{x}$ alloys decreases with the $\mathrm{Cu}$ addition. Though we find that $\mathrm{Cu}$ doping shift the MT temperature to 
higher temperature, the $T_{\mathrm{c}}$ changes in the opposite directions. Magnetic-field-induced martensitic transformation indicates a transformation sequence of ferromagnetic martensite, ferromagnetic austenite and paramagnetic austenite upon heating. The $T_{\mathrm{c}}$ of austenite is higher than MT temperature is essential to using Magnetic-field-induced martensitic transformation. Therefore, it is necessary to study how to synchronously increase the $T_{\mathrm{c}}$ and MT temperature in the further studies.

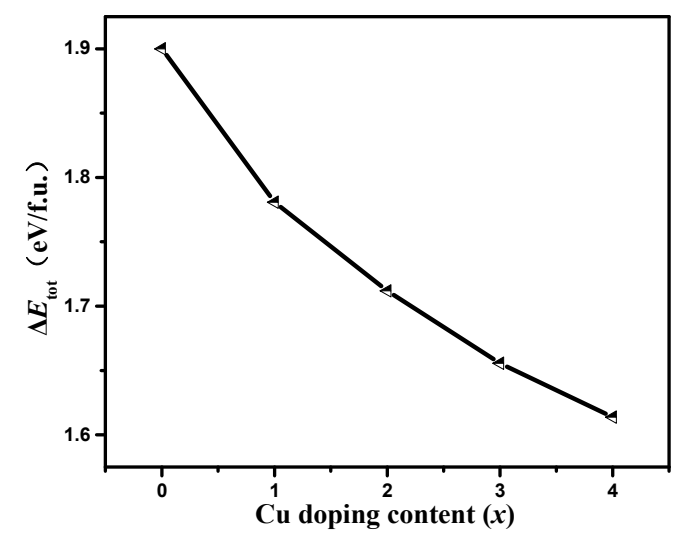

Figure 6. The energy difference between the paramagnetic austensite and ferromagnetic austenite per formula unit for $\mathrm{Ni}_{16} \mathrm{Mn}_{12} \mathrm{Sn}_{4-x} \mathrm{Cu}_{x}$ alloys ( $x=0,1,2,3$, and 4).

In order to have a further insight into the electronic structure, the magnetic properties, and the martensitic transformation of $\mathrm{Ni}_{16} \mathrm{Mn}_{12} \mathrm{Sn}_{4-x} \mathrm{Cu}_{x}$ alloys $(x=0,1$, and 2$)$, we investigated the total density of states (DOS) for both the austenite (as indicated by the black line in Figure 7) and martensite phases (as indicated by the red line in Figure 7). It can be seen from Figure 7 that, in the down-spin DOS of the austenite phase, there exists a sharp DOS peak at the Fermi level, indicating the bonding character between the atoms in $\mathrm{Ni}_{16} \mathrm{Mn}_{12} \mathrm{Sn}_{4-x} \mathrm{Cu}_{x}$ around the Fermi level, and this is apparently responsible for the martensitic transformation. However, the DOS peak at the Fermi level $\left(\mathrm{E}_{\mathrm{f}}\right)$ almost disappears when the transition of the tetragonal martensite phase to/from the cubic austenite phase takes place, as seen from the inset (expanded the regions near $\mathrm{E}_{\mathrm{f}}$ ). This behavior suggests that the DOS in the vicinity of the $\mathrm{E}_{\mathrm{f}}$ is an important factor that triggers the occurrence of martensitic transformation [31].

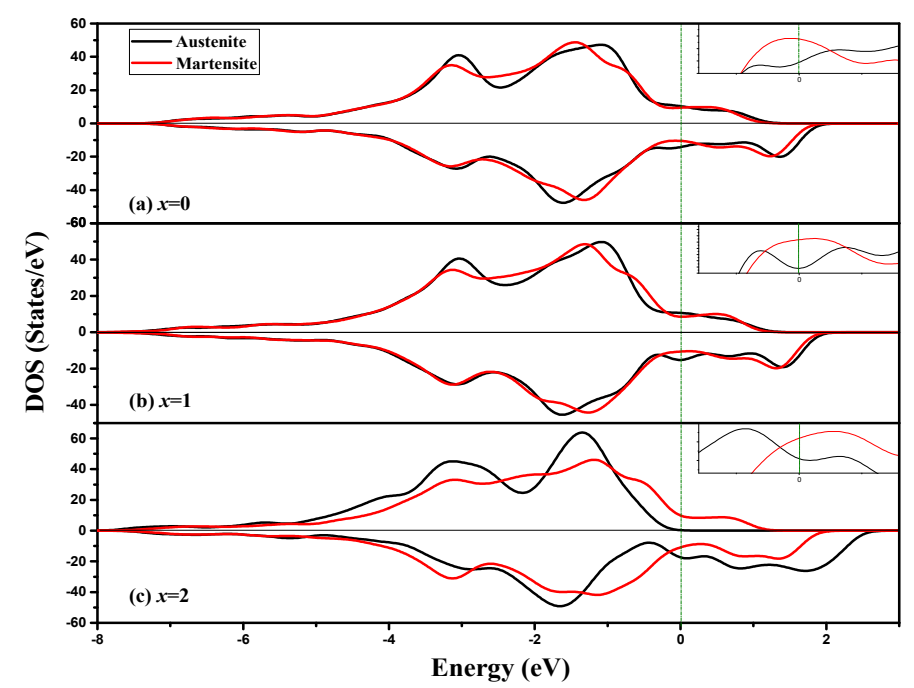

Figure 7. The total dos plots for the $\mathrm{Ni}_{16} \mathrm{Mn}_{12} \mathrm{Sn}_{4-x} \mathrm{Cu}_{x}(x=0,1,2)$ for both cubic austenite and tetragonal martensite phase. Each phase is consisting of the up-spin and sown-spin channels. The vertical dashed line is chosen as Fermi level $\mathrm{E}_{\mathrm{f}}$. The regions near the Fermi level are shown in an expanded scale in the inset. 
The aforementioned results suggest that Mn-Mn magnetic interaction in the cubic phase of $\mathrm{Ni}-\mathrm{Mn}$-Sn alloy has been turned from anti-ferromagnetism to ferromagnetism by the substitution of $\mathrm{Cu}$ for $\mathrm{Sn}$. To gain insight into the origin of magnetic properties and magnetostructural transformation in Ni-Mn-Sn-Cu, we have investigated the electronic structure. Also, because the trend of each change in other specimens is similar to the case of $x=2$, here we only choose the case of $x=0$ and 2 for comparison. Figure 8 illustrates the DOS for the cubic and martensitic phase of $\mathrm{Ni}_{16} \mathrm{Mn}_{12} \mathrm{Sn}_{4-x} \mathrm{Cu}_{x}$ alloys ( $x=0$, and 2). In the case of $x=0, \mathrm{Mn} 3 \mathrm{~d}$ state of majority and minority spin are split with respect to the Fermi level. This distribution makes Mn mostly responsible for the magnetization. Moreover, it can be noted that the magnetic moment of $\mathrm{Mn}_{\mathrm{Sn}}$ is anti-parallel to that of $\mathrm{Mn}_{\mathrm{Mn}}$ (as indicated by the arrow). Ni $3 \mathrm{~d}$ states have similar distribution in the majority and minority spin, resulting in a rather small magnetization. With $\mathrm{Cu}$ addition, the $\mathrm{Mn}_{\mathrm{Mn}} 3 \mathrm{~d}$ state is similar to that of before. However, $\mathrm{Mn}_{\mathrm{Sn}} 3 \mathrm{~d}$ state is altered. $\mathrm{Mn}_{\mathrm{Sn}} 3 \mathrm{~d}$ state of majority spin is mostly below the Fermi level, while that of minority spin is mostly above the Fermi level. Thus, the magnetic moment of $\mathrm{Mn}_{\mathrm{Sn}}$ is parallel to that of $\mathrm{Mn}_{\mathrm{Mn}}$, giving rise to the enhancement of the total magnetic moment. This is in agreement with the aforementioned results. This result further clarifies the change of magnetic moment of Ni-Mn-Sn-Cu.
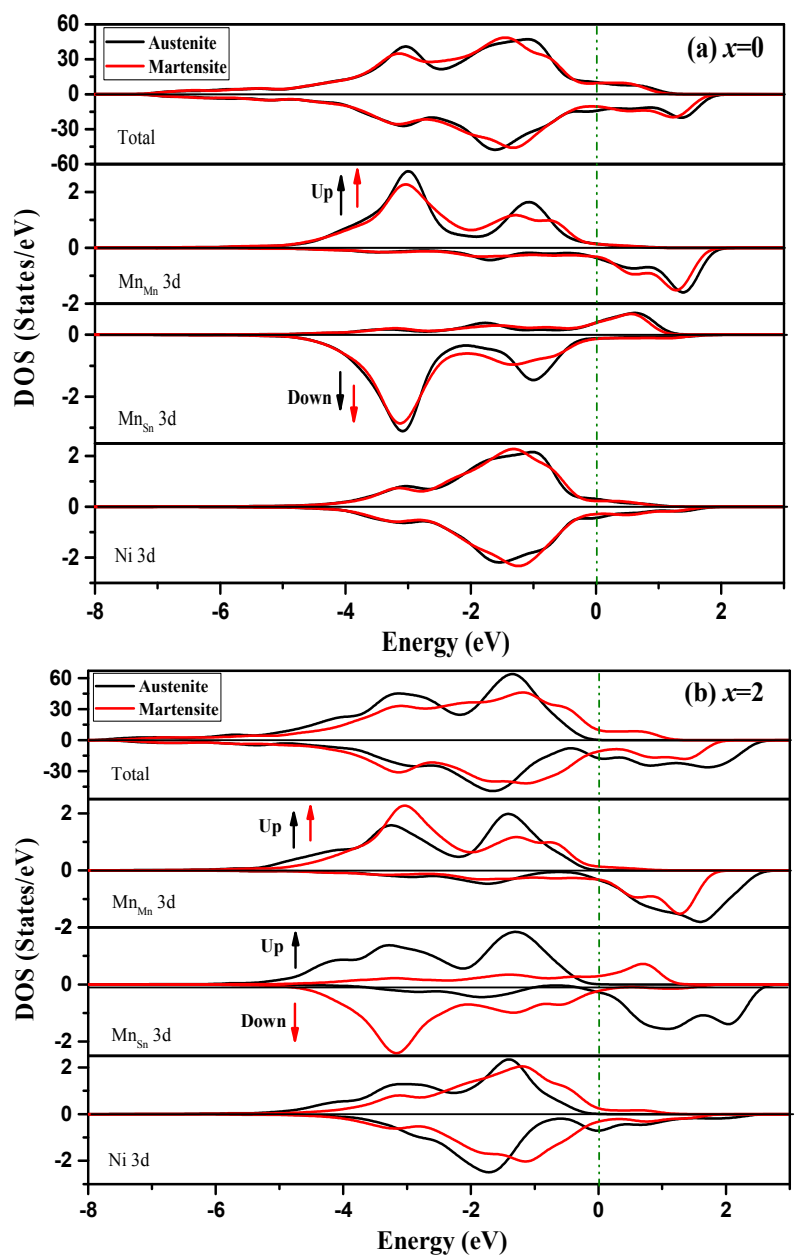

Figure 8. The partial density of states (DOS) plots for $\mathrm{Ni}_{16} \mathrm{Mn}_{12} \mathrm{Sn}_{4-x} \mathrm{Cu}_{x}$ alloys in both cubic austenite and tetragonal martensite phase, (a) $x=0$; and (b) $x=2$. The vertical dashed line is chosen as the Fermi level $\mathrm{E}_{\mathrm{f}}$.

\section{Conclusions}

In summary, in order to design the new magnetic shape memory alloy material which can show a large $\Delta M$ and a higher MT temperature, first principle electronic structure calculations were 
employed. We have studied the total energy, the magnetic properties, and the magnetic-field-induced martensitic transformation for $\mathrm{Ni}_{16} \mathrm{Mn}_{12} \mathrm{Sn}_{4-x} \mathrm{Cu}_{x}$ alloys $(x=0,1,2,3$, and 4). The equilibrium equation of state results shows that antiferromagnetic cubic austenite becomes ferromagnetic cubic austenite in Ni-Mn-Sn alloys by $\mathrm{Cu}$ addition. Especially in the case of $x=2$, drastic changes in the magnetic properties are expected to occur between the ferromagnetic austensite $(6.74 \mu \mathrm{B} / \mathrm{f}$.u.) and the antiferromagnetic martensite phase $(1.98 \mu \mathrm{B} / \mathrm{f}$.u.). This directly leads to the large $\Delta M$ and enhances the MFIRMT. Meanwhile, the martensitic transformation shifts to the higher temperature, owing to the energy difference between the austenite $L 2_{1}$ structure, and the tetragonal martensite $L 1_{0}$ structure increases by $\mathrm{Cu}$ doping. The calculations mentioned above may help in gaining an insight into the doping of $\mathrm{Cu}$ behavior in Ni-Mn-Sn and provide guidance to the new material design. Furthermore, the total energy difference between paramagnetic and ferromagnetic austenite indicates the decrease in $T_{\mathrm{c}}$ with $\mathrm{Cu}$ addition. We expect that the restriction would be resolved in the further studies.

Acknowledgments: The authors acknowledge the supports of National Natural Science Foundation of China (Grant Nos. 51471064, 51301054 and 50901026); and Program for Youth Academic Backbone in Heilongjiang Provincial University (Grant No. 1251G022); and Scientific Research Fund of Heilongiiang Provincial Education Department (No. 12541138).

Author Contributions: Kun Zhang conceived and designed the experiments; Xiaohua Tian, Kun Zhang and Wenbin Zhao performed the experiments; Changlong Tan, Erjun Guo, and Xiaohua Tian analyzed the data; Kun Zhang, Changlong Tan, Xiaohua Tian, and Wei Cai wrote the paper.

Conflicts of Interest: The authors declare no conflict of interest.

\section{References}

1. Ullakko, K.; Huang, J.K.; Kantner, C.; O’Handley, R.C.; Kokorin, V.V. Large magnetic-field-induced strains in $\mathrm{Ni}_{2} \mathrm{MnGa}$ single crystals. Appl. Phys. Lett. 1996, 69, 1966-1968. [CrossRef]

2. Sutou, Y.; Imano, Y.; Koeda, N.; Omori, T.; Kainuma, R.; Ishida, K.; Oikawa, K. Magnetic and martensitic transformations of $\mathrm{NiMnX}(X=\mathrm{In}, \mathrm{Sn}, \mathrm{Sb})$ ferromagnetic shape memory alloys. Appl. Phys. Lett. 2004, 85, 4358. [CrossRef]

3. Murray, S.J.; Marioni, M.; Allen, S.M.; O’Handley, R.C.; Lograsso, T.A. 6\% magnetic-field-induced strain by twin-boundary motion in ferromagnetic Ni-Mn-Ga. Appl. Phys. Lett. 2000, 77, 886-888. [CrossRef]

4. Kainuma, R.; Imano, Y.; Ito, W.; Sutou, Y.; Morito, H.; Okamoto, S. Magnetic-field-induced shape recovery by reverse phase transformation. Nature 2006, 439, 957-960. [CrossRef] [PubMed]

5. Sozinov, A.; Soroka, A.; Lanska, N.; Rameš, M.; Straka, L.; Ullakko, K. Temperature dependence of twinning and magnetic stresses in $\mathrm{Ni}_{46} \mathrm{Mn}_{24} \mathrm{Ga}_{22} \mathrm{Co}_{4} \mathrm{Cu}_{4}$ alloy with giant $12 \%$ magnetic field-induced strain. Scr. Mater. 2017, 131, 33-36. [CrossRef]

6. Sozinov, A.; Likhachev, A.A.; Lanska, N.; Ullakko, K. Giant magnetic-field-induced strain in NiMnGa seven-layered martensitic phase. Appl. Phys. Lett. 2002, 80, 1746-1748. [CrossRef]

7. Karaca, H.E.; Karaman, I.; Basaran, B.; Chumlyakov, Y.J.; Maier, H.J. Magnetic field and stress induced martensite reorientation in NiMnGa ferromagnetic shape memory alloy single crystals. Acta Mater. 2006, 54, 233-245. [CrossRef]

8. Karaca, H.E.; Karaman, I.; Basaran, B.; Ren, Y.; Chumlyakov, Y.J.; Maier, H.J. Magnetic Field-Induced Phase Transformation in NiMnCoIn Magnetic Shape-Memory Alloys-A New Actuation Mechanism with Large Work Output. Adv. Funct. Mater. 2009, 19, 983-998. [CrossRef]

9. Zhao, D.; Liu, J.; Chen, X.; Sun, W.; Li, Y.; Zhang, M. Giant caloric effect of low-hysteresis metamagnetic shape memory alloys with exceptional cyclic functionality. Acta Mater 2017, 133, 217-223. [CrossRef]

10. Yang, Y.B.; Ma, X.B.; Chen, X.G.; Wei, J.Z.; Wu, R.; Han, J.Z.; Du, H.L.; Wang, C.S.; Liu, S.Q.; Yang, Y.C.; et al. Structure and exchange bias of $\mathrm{Ni}_{50} \mathrm{Mn}_{37} \mathrm{Sn}_{13}$ ribbons. J. Appl. Phys. 2012, 111, 07A916. [CrossRef]

11. Han, Z.D.; Wang, D.H.; Zhang, C.L.; Xuan, H.C.; Gu, B.X.; Du, Y.W. Low-field inverse magnetocaloric effect in $\mathrm{Ni}_{50-x} \mathrm{Mn}_{39+x} \mathrm{Sn}_{11}$ Heusler alloys. Appl. Phys. Lett. 2007, 90, 042507. [CrossRef]

12. Koyama, K.; Watanabe, K.; Kanomata, T.; Kainuma, R.; Oikawa, K.; Ishida, K. Observation of field-induced reverse transformation in ferromagnetic shape memory alloy $\mathrm{Ni}_{50} \mathrm{Mn}_{36} \mathrm{Sn}_{14}$. Appl. Phys. Lett. 2006, 88, 132505. [CrossRef] 
13. Liu, J.; Gottschall, T.; Skokov, K.P.; Moore, J.D.; Gutfleisch, O. Giant magnetocaloric effect driven by structural transitions. Nat. Mater. 2012, 11, 620-626. [CrossRef] [PubMed]

14. Raji, G.R.; Uthaman, B.; Thomas, S.; Suresh, K.G.; Varma, M.R. Magnetocaloric properties, exchange bias, and critical behavior of Ge substituted $\mathrm{Ni}_{50} \mathrm{Mn}_{36} \mathrm{Sn}_{14}$ Heusler alloys. J. Appl. Phys. 2015, 117, 103908. [CrossRef]

15. Nayak, A.K.; Suresh, K.G.; Nigam, A.K. Irreversibility of field-induced magnetostructural transition in $\mathrm{NiCoMnSb}$ shape memory alloy revealed by magnetization, transport and heat capacity studies. Appl. Phys. Lett. 2010, 96, 112503. [CrossRef]

16. Rama Rao, N.V.; Chandrasekaran, V.; Suresh, K.G. Effect of Ni/Mn ratio on phase transformation and magnetic properties in Ni-Mn-In alloys. J. Appl. Phys. 2010, 108, 043913. [CrossRef]

17. Xuan, H.C.; Wang, D.H.; Zhang, C.L.; Han, Z.D.; Gu, B.X.; Du, Y.W. Boron's effect on martensitic transformation and magnetocaloric effect in $\mathrm{Ni}_{43} \mathrm{Mn}_{46} \mathrm{Sn}_{11} \mathrm{~B}_{x}$ alloys. Appl. Phys. Lett. 2008, 92, 102503. [CrossRef]

18. Esakki Muthu, S.; Rama Rao, N.V.; Thiyagarajan, R.; Devarajan, U.; Manivel Raja, M.; Arumugam, S. Influence of chemical substitution, magnetic field, and hydrostatic pressure effect on martensitic and intermartensitic transition in bulk $\mathrm{Ni}_{49-x} \mathrm{Cu}_{x} \mathrm{Mn}_{38} \mathrm{Sn}_{13}(0.5 \leq x \leq 2)$ Heusler alloys. Appl. Phys. Lett. 2014, 104, 092404. [CrossRef]

19. Chattopadhyay, M.K.; Sharma, V.K.; Chouhan, A.; Arora, P.; Roy, S.B. Combined effect of hydrostatic pressure and magnetic field on the martensitic transition in the $\mathrm{Ni}_{49} \mathrm{CuMn}_{34} \mathrm{In}_{16}$ alloy. Phys. Rev. B 2011, 84, 064417. [CrossRef]

20. Feng, L.; Ma, L.; Liu, E.K.; Wu, G.H.; Wang, W.H.; Zhang, W.X. Magnetic-field-induced martensitic transformation in MnNiAl: Co alloys. Appl. Phys. Lett. 2012, 100, 152401. [CrossRef]

21. Castillovilla, P.O.; Mañosa, L.; Planes, A.; Sotoparra, D.E.; Sánchezllamazares, J.L.; Floreszúñiga, H. Elastocaloric and magnetocaloric effects in Ni-Mn-Sn(Cu) shape-memory alloy. J. Appl. Phys. 2013, 113, 053506. [CrossRef]

22. Li, Y.; Sun, W.; Zhao, D.; Xu, H.; Liu, J. An $8 \mathrm{k}$ elastocaloric temperature change induced by $1.3 \%$ transformation strain in $\mathrm{Ni}_{44} \mathrm{Mn}_{45-x} \mathrm{Sn}_{11} \mathrm{Cu}_{x}$ alloys. Scr. Mater. 2017, 130, 278-282. [CrossRef]

23. Wang, D.H.; Zhang, C.L.; Xuan, H.C.; Han, Z.D.; Zhang, J.R.; Tang, S.L. The study of low-field positive and negative magnetic entropy changes in $\mathrm{Ni}_{43} \mathrm{Mn}_{46-x} \mathrm{Cu}_{x} \mathrm{Sn}_{11}$ alloys. J. Appl. Phys. 2007, 102, 013909. [CrossRef]

24. Xuan, H.C.; Han, P.D.; Wang, D.H.; Du, Y.W. Magnetic and magnetocaloric properties in Cu-doped high Mn content $\mathrm{Mn}_{50} \mathrm{Ni}_{40-x} \mathrm{Cu}_{x} \mathrm{Sn}_{10}$ heusler alloys. Intermetallics 2014, 54, 120-124. [CrossRef]

25. Wang, X.; Shang, J.X.; Wang, F.H.; Jiang, C.B.; Xu, H.B. Effect of $3 d$ transition elements substitution for Ni in $\mathrm{Ni}_{2} \mathrm{Mn}_{1+x} \mathrm{Sn}_{1-x}$ on the phase stability and magnetic properties: A first principle investigation. J. Magn. Magn. Mater. 2014, 368, 286-294. [CrossRef]

26. Segall, M.; Lindan, P.; Probet, M.; Pickard, C.; Hasnip, P.; Clark, S.; Payne, M. First-principles simulation: ideas, illustrations and the CASTEP code. J. Phys. Condens. Matter 2002, 14, 2717-2744.

27. Vanderbilt, D. Soft self-consistent pseudopotentials in a generalized eigenvalue formalism. Phys. Rev. B 1990, 41, 7892-7895. [CrossRef]

28. Li, C.M.; Luo, H.B.; Hu, Q.M.; Yang, R.; Johansson, B.; Vitos, L. First-principles investigation of the composition dependent properties of $\mathrm{Ni}_{2+x} \mathrm{Mn}_{1-x}$ Ga shape-memory alloys. Phys. Rev. B 2010, 82, 2181-2188.

29. Bai, J.; Raulot, J.M.; Zhang, Y.; Esling, C. The effects of alloying element co on Ni-Mn-Ga ferromagnetic shape memory alloys from first-principles calculations. Appl. Phys. Lett. 2011, 98, 1641033. [CrossRef]

30. Sato, K.; Dederichs, P.H.; Katayama-Yoshida, H. Curie temperatures of III-V diluted magnetic semiconductors calculated from first principles. Europhys. Lett. 2003, 61, 403-408. [CrossRef]

31. Tan, C.L.; Huang, Y.W.; Tian, X.H.; Jiang, J.X.; Cai, W. Origin of magnetic properties and martensitic transformation of Ni-Mn-In magnetic shape memory alloys. Appl. Phys. Lett. 2012, 100, 132402. [CrossRef]

(C) 2018 by the authors. Licensee MDPI, Basel, Switzerland. This article is an open access article distributed under the terms and conditions of the Creative Commons Attribution (CC BY) license (http://creativecommons.org/licenses/by/4.0/). 